\title{
Induction of cell cycle arrest via the p21, p27-cyclin E,A/Cdk2 pathway in SMMC-7721 hepatoma cells by clioquinol
}

\author{
ZHIWEI HUANG ${ }^{1,2^{*}}$ \\ LIANQIU WANG ${ }^{1}$ \\ LIFENG CHEN ${ }^{1,3}$ \\ YIFEI ZHANG ${ }^{3}$ \\ PING SHI ${ }^{3 *}$ \\ ${ }^{1}$ Key Lab of Eco-Textile (Ministry of \\ Education), College of Chemistry \\ Chemical Engineering and Biotechnology \\ Donghua University \\ Shanghai 201620, China \\ ${ }^{2}$ Key Laboratory of Organofluorine \\ Chemistry, Shanghai Institute of \\ Organic Chemistry, Chinese Academy \\ of Sciences, Shanghai 200032, China \\ ${ }^{3}$ State Key Laboratory of Bioreactor \\ Engineering, East China University of \\ Science and Technology \\ Shanghai 200237, China
}

\begin{abstract}
Clioquinol has been shown to have anticancer activity in several carcinoma cells. In this study, we preliminarily examined the effect of clioquinol in human SMMC-7721 hepatoma and QSG-7701 normal hepatic cells. Our results indicated that clioquinol did not significantly affect survival of QSG-7701 cells, whereas it reduced cell viability in a concentration- and time-dependent manner in SMMC-7721 cells. Clioquinol did not trigger autophagy and apoptosis, while it induced cell cycle arrest in the S-phase in SMMC7721 cells. Additionally, down-regulation of cyclin D1, A2, E1, Cdk2 and up-regulation of p21, p27 were detected after the treatment with clioquinol. The results demonstrated for the first time that clioquinol suppressed cell cycle progression in the S-phase in SMMC-7721 cells via the p21, p27-cyclin E,A/Cdk2 pathway. This suggests that clioquinol may have a therapeutic potential as an anticancer drug for certain malignances.
\end{abstract}

Keywords: clioquinol, human SMMC-7721 hepatoma cells, cell cycle S-phase arrest, cyclin E/A-Cdk2

Accepted July 14, 2015

Clioquinol (5-chloro-7-iodoquinolin-8-ol), a quinoline compound, acts as a mild metal chelator and/or ionophore, binding metals like copper and zinc. It has been used as an antibacterial drug for treating diarrhea and skin infections (1). However, clioquinol was withdrawn from the market due to subacute myelo-optic neuropathy (SMON) in Japan (2, 3). In recent years, toxicology studies of clioquinol performed in both animals and humans show it has low side effects $(4,5)$. Owing to its ability to bind copper/zinc and dissolve beta-amyloid plaques by activating autophagy in the brain, clioquinol has re-emerged as a potential therapy for Alzheimer's disease $(6,7)$. Clioquinol was also reported to have anticancer activity in several carcinoma cells (8-11). Especially, in preclinical models of hematologic malignancies and solid tumors, clioquinol displays anticancer efficacy in vitro and in vivo (12-14). Thus, clioquinol may be a novel anti-cancer agent. However, pharmacological actions of clioquinol on cancer cells still remain unclear.

\footnotetext{
*Correspondence; e-mail: zhiweih@dhu.edu.cn, ship@ecust.edu.cn
} 
In the present study, we explored the anti-cancer effects of clioquinol on human SMMC-7721 hepatoma cells.

\section{EXPERIMENTAL}

\section{Reagents and antibodies}

Clioquinol was obtained from Tokyo KaSei Industry Co., Ltd. (Japan) and dissolved in DMSO. Cell counting kit-8 was purchased from Dojindo molecular technologies Inc. (Dojindo, Japan). Propidium iodine (PI), Hoechst 33258 and Acridine Orange (AO) were purchased from Sigma-Aldrich (USA). Annexin V-FITC apoptosis detection kit was purchased from (Molecular Biology \& Chemistry, China). ECL Western blotting detection reagents were purchased from GE Healthcare (UK). Other reagents used in the study were purchased from Sinopharm Chemical Reagent Co., Ltd. (China).

P21, P27, cyclin A, cyclin E and Cdk2 rabbit monoclonal antibodies were purchased from Epitomics Inc. (USA). HRP-conjugated monoclonal mouse anti-glyceraldehyde-3-phosphate dehydrogease (GAPDH) was purchased from Kangchen Biotech (China). The secondary antibody HRP-labeled goat anti-rabbit IgG $(\mathrm{H}+\mathrm{L})$ was purchased from Novoprotein Scientific Inc. (China).

\section{Cell line and cell culture}

Human hepatoma cell line SMMC-7721 and immortal hepatocytes QSG-7701 were supplied by the Type Culture Collection of the Chinese Academy of Sciences (China). Cells were cultured at $37^{\circ} \mathrm{C}$ under a humidified $5 \% \mathrm{CO}_{2}$ atmosphere in DMEM and RPMI-1640 medium supplemented with $10 \%$ fetal bovine serum (FBS) (GIBCO, USA) and $1 \%$ penicillin-streptomycin $\left(10,000 \mathrm{U} \mathrm{mL}^{-1}\right.$ penicillin and $10 \mathrm{mg} \mathrm{mL}^{-1}$ streptomycin (Solarbio Life Science, China).

\section{Cell viability and apoptosis assay}

Cell viability was evaluated by Cell counting kit-8 according to the manufacturer's instructions. Cells were plated in 96-well micro-well plates in $0.1 \mathrm{~mL}$ medium, at a density of $1 \times 10^{5}$ cells per well. The cells were incubated without clioquinol ( $0.1 \%$ DMSO) or with $1,2,5 \mu \mathrm{mol} \mathrm{L}{ }^{-1}$ clioquinol solution. Absorbance was measured at $450 \mathrm{~nm}$ with a Genios multifunction-reader (Tecan GENios Pro, Tecan Group Ltd., Switzerland). Apoptosis was examined using flow cytometry (FACScalibur, BD Bioscenices, USA) after staining the cells with annexin V-FITC and propidium iodide (PI) (Majorbio Bio-Pharm Technology Co., China). Data analysis was performed with standard CellQuest software (BD FACScan ${ }^{\mathrm{TM}}$, USA).

\section{Staining}

Hoechst 33258 staining. - SMMC-7721 cells at $2 \times 10^{5}$ cells $\mathrm{mL}^{-1}$ were seeded in 24 -well plates and treated with $5 \mu \mathrm{mol} \mathrm{L}^{-1}$ clioquinol or $0.1 \%$ DMSO for $72 \mathrm{~h}$. The cells were fixed in $4 \%$ paraformaldehyde at $4{ }^{\circ} \mathrm{C}$ for $30 \mathrm{~min}$, and then stained with $5 \mu \mathrm{g} \mathrm{mL} \mathrm{m}^{-1}$ Hoechst 33258 , a DNA-specific fluorescent dye, for $10 \mathrm{~min}$ at $37^{\circ} \mathrm{C}$. The stained cells were observed under a fluorescence microscope (Olympus BX51, Japan). 
Vital cell staining with acridine orange for autophagy detection. - Cells were seeded on slides and staining with acridine orange was performed according to published procedures (15), adding a final concentration of $1 \mu \mathrm{g} \mathrm{mL} \mathrm{m}^{-1}$ acridine orange for $30 \mathrm{~min}$ at $37^{\circ} \mathrm{C}$. Photographs were obtained with a fluorescence microscope (200×) (Olympus BX51).

\section{Cell cycle analysis}

Cellular DNA content was determined by flow cytometry. After incubation with 5 $\mu \mathrm{mol} \mathrm{L}-1$ or $0.1 \%$ DMSO for 48 and $72 \mathrm{~h}$, cells were harvested, washed twice with ice-cold PBS, fixed with $70 \%$ ethanol for $18 \mathrm{~h}$ at $4{ }^{\circ} \mathrm{C}$. Cells were then washed twice with ice-cold PBS and re-suspended in PBS. RNA was digested with RNase A $\left(10 \mu \mathrm{g} \mathrm{mL}{ }^{-1}\right)$ and DNA was stained with PI $\left(10 \mu \mathrm{g} \mathrm{mL}^{-1}\right)$. Stained cells were analyzed with a FACScan flow cytometer (Becton Dickinson, USA). Cell cycle distribution was analyzed by measuring cell fractions in $G_{0} / G_{1}, S$, and $G_{2} / M$ phases using ModFit LT 2.0 software (Verity Software, Topsham, USA). Results shown are representative of three independent experiments.

\section{Real-time PCR}

The cells were incubated with $5 \mu \mathrm{mol} \mathrm{L}{ }^{-1}$ clioquinol or $0.1 \%$ DMSO for $72 \mathrm{~h}$. Total RNA extraction and complementary DNA (cDNA) preparation were performed using TRNzol reagent and FastQuant RT kit (with gDNase, Tiangen, China), respectively. The real-time PCR analysis was performed in triplicate using SuperReal PreMix Plus (SYBR Green) (Tiangen, China) in a CFX96 Real-Time PCR Detection System (Bio-Rad, USA). The reactions were as follows: activation of the Taq DNA polymerase at $95{ }^{\circ} \mathrm{C}$ for $15 \mathrm{~min}$ and 40 cycles of $95^{\circ} \mathrm{C}$ for $10 \mathrm{~s}$ and $60^{\circ} \mathrm{C}$ for $32 \mathrm{~s}$. The primers are listed in Table I. Gapdh was selected as internal control. The relative changes in gene expression were calculated using the equation by Livak (16):

$$
2^{-\Delta \Delta \mathrm{CT}} \text {, where } \Delta \Delta C_{\mathrm{T}}=\left(C_{\mathrm{T}, \text { Target }}-C_{\mathrm{T}, \text { Gapdh }}\right)_{\text {Treatment }}-\left(C_{\mathrm{T}, \text { Target }}-C_{\mathrm{T}, \text { Gapdh }}\right)_{\mathrm{Control}}
$$

$C_{\mathrm{T}, \text { Target }}$ is the threshold cycle for internal reference Gapdh gene. The treatment and control mean the cells treated with and without clioquinol, respectively. Data were expressed as a fold change compared to control samples (mean \pm SD of three replicates).

Table I. Primer sequences used in real-time PCR analysis

\begin{tabular}{lll}
\hline Gene name & Left primer & Right primer \\
\hline Cyclin A2 & TGGAAAGCAAACAGTAAACAGCC & GGGCATCTTCACGCTCTATTT \\
Cyclin D1 & CCGTCCATGCGGAAGATC & ATGGCCAGCGGGAAGAC \\
Cyclin E1 & GCCAGCCTTGGGACAATAATG & AGTTTGGGTAAACCCGGTCAT \\
Cdk2 & CCAGGAGTTACTTCTATGCCTGA & TTCATCCAGGGGAGGTACAAC \\
p21 & CGATGGAACTTCGACTTTGTCA & GCACAAGGGTACAAGACAGTG \\
p27 & AACGTGCGAGTGTCTAACGG & CCAGGAGTTACTTCTATGCCTGA \\
Gapdh & AGAAGGCTGGGGCTCATTTG & AGGGGCCATCCACAGTCTTC \\
\hline
\end{tabular}




\section{Western blot}

The cells were incubated with $5 \mu \mathrm{mol} \mathrm{L}-1$ clioquinol or $0.1 \%$ DMSO for $72 \mathrm{~h}$. Cell lysates were obtained using standard techniques and RIPA lysis buffer (Beyotime Institute of Biotechnology, China). Protein concentration was determined by the Bradford protein assay (Bio-Rad, USA). A quantity of $50 \mu \mathrm{g}$ total protein per lane was separated by $12 \%$ sodium dodecyl sulfate-polyacrylamide gel electrophoresis (SDS-PAGE) and transferred to Amersham Hybond-P polyvinylidene fluoride (PVDF) membranes (GE Healthcare). Membranes were blocked with $5 \%$ milk powder in $0.05 \%$ Tween-Tris buffer saline (TBS), incubated with specific primary antibodies and then with the appropriate horseradish peroxidase-conjugated secondary antibodies (Sinbio, China). GAPDH was used as an internal standard. Detection of the target proteins on the membranes was performed using the ECL western blotting detection reagents (GE Healthcare, UK). Signal density was detected using the MF-ChemiBIS family including gel capture software (DNR Bio-Image system, Ltd., Israel) and quantitatively analyzed by densitometry using the Image J software.

\section{Statistical analysis}

The experiments were repeated three times. The data are presented as the mean \pm SD (for each group). Statistical analysis was performed using Student's $t$-test (paired). Values of $p<0.05$ were considered to be statistically significant.

\section{RESULTS AND DISCUSSION}

\section{Clioquinol causes SMMC-7721 cell death}

Using the WST-8 based colorimetric assay cell counting kit 8, the effects of clioquinol on cell viability were measured. As shown in Fig. 1a, clioquinol induced a time- and concentration-dependent decrease in the viability of SMMC-7721 cells. After 72 hours of treatment, the half maximal inhibitory concentration $\left(I C_{50}\right)$ of clioquinol was $1.0 \mu \mathrm{mol} \mathrm{L}^{-1}$. It is noteworthy that clioquinol did not induce significant decrease in the viability of normal human liver cells QSG-7701 (Fig. 1b). Obviously, the cell growth inhibitory effect of clioquinol on the human hepatoma SMMC-7721cells was stronger than that on the normal liver QSG-7701cells. In addition, the highest concentration of clioquinol (5 $\mu \mathrm{mol}$ $\mathrm{L}^{-1}$ ) in our study did not exhibit significant cytotoxicity in QSG-7701 cells. The results further confirmed that clioquinol has low side effects (8-11).

\section{Clioquinol does not induce cell apoptosis and autophagy}

We sought to explore the possible inhibition of SMMC-7721 cell growth. Annexin V-FITC and PI staining assay were used to detect cell apoptosis. No significant induction of apoptosis occurred in SMMC-7721 cells after 48-h treatment with $5 \mu \mathrm{mol} \mathrm{L}{ }^{-1}$ clioquinol (data not shown). Then, Hoechst 33258, a DNA-specific fluorescent dye, was chosen to observe the morphologic characteristics of apoptosis in SMMC-7721 cells. As shown in Fig. 2 , no apoptotic cells could be found after the clioquinol treatment for $72 \mathrm{~h}$ by fluorescence 

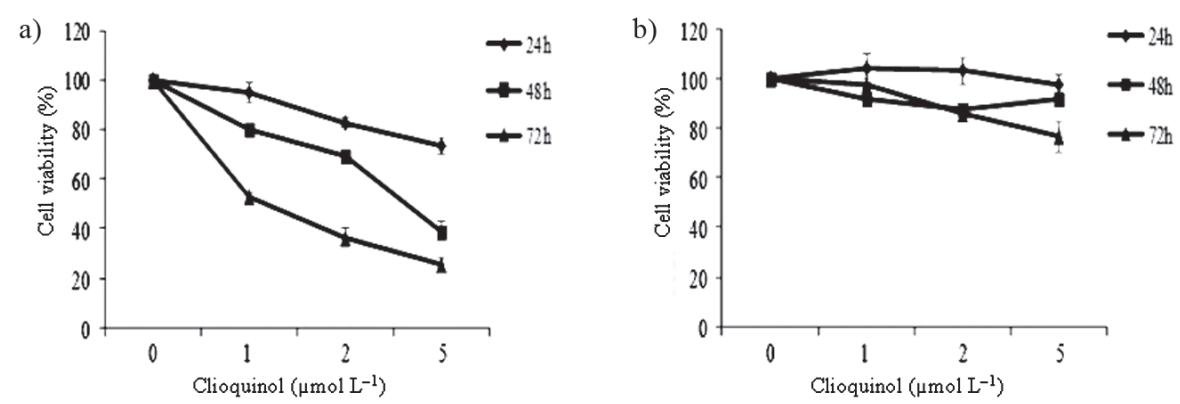

Fig. 1. The inhibitory effect of clioquinol on the: a) viability of SMMC-7721 cells and b) QSG-7701 cells.

microscopy. The above observations indicate that apoptosis is not the cause of cell death induced by clioquinol. We then questioned if it induced autophagy, another form of cell death, in SMMC-7721 cells. To address this issue, vital staining of cells treated with clioquinol for $72 \mathrm{~h}$ was performed with acridine orange, a selective fluorescent marker of autophagic vesicles. As shown in Figure 2, no acidic vesicles were observed. Likewise, clioquinol did not induce autophagy in SMMC-7721 cells.

\section{Cell cycle arrest is induced by clioquinol}

To examine whether clioquinol-induced growth inhibition was associated with cell cycle regulation, the cell cycle distribution was analyzed by flow cytometry. After SMMC7721 cells were incubated with clioquinol for 48 and $72 \mathrm{~h}$, cells were harvested and further prepared for cell cycle analysis. Figs. $3 a$ and $b$ showed that clioquinol treatments for 48 and $72 \mathrm{~h}$ resulted in accumulation of SMMC-7721 cells in the S phase. Cell populations in the

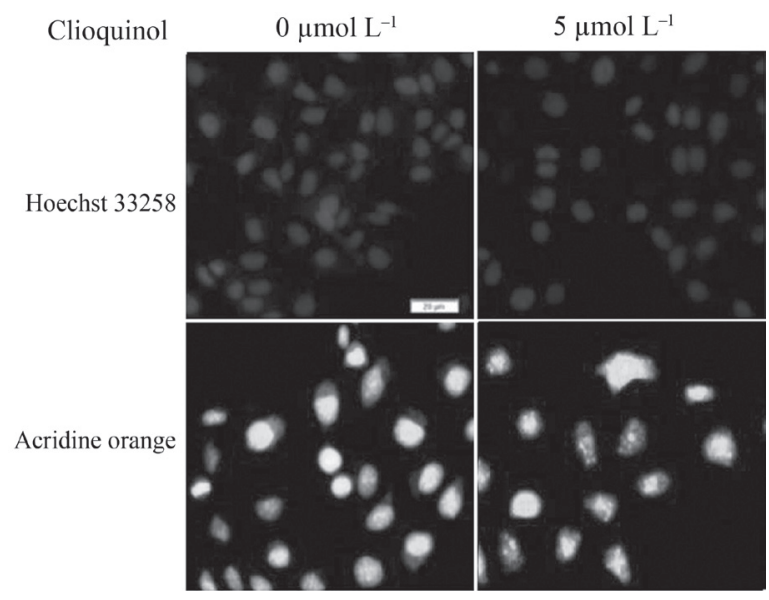

Fig. 2. Analysis of apoptosis and autophagy after clioquinol treatment in SMMC-7721 cells. 
S phase were $35.2 \%$ and $36.6 \%$ in control SMMC-7721 cells. However, after 48 and $72 \mathrm{~h}$ of incubation with $5 \mu \mathrm{mol} \mathrm{L}^{-1}$ clioquinol, the $\mathrm{S}$ population was noticeably enhanced by 44.8 and $58.2 \%$, respectively $(p<0.05)$ (Fig. 3 ).
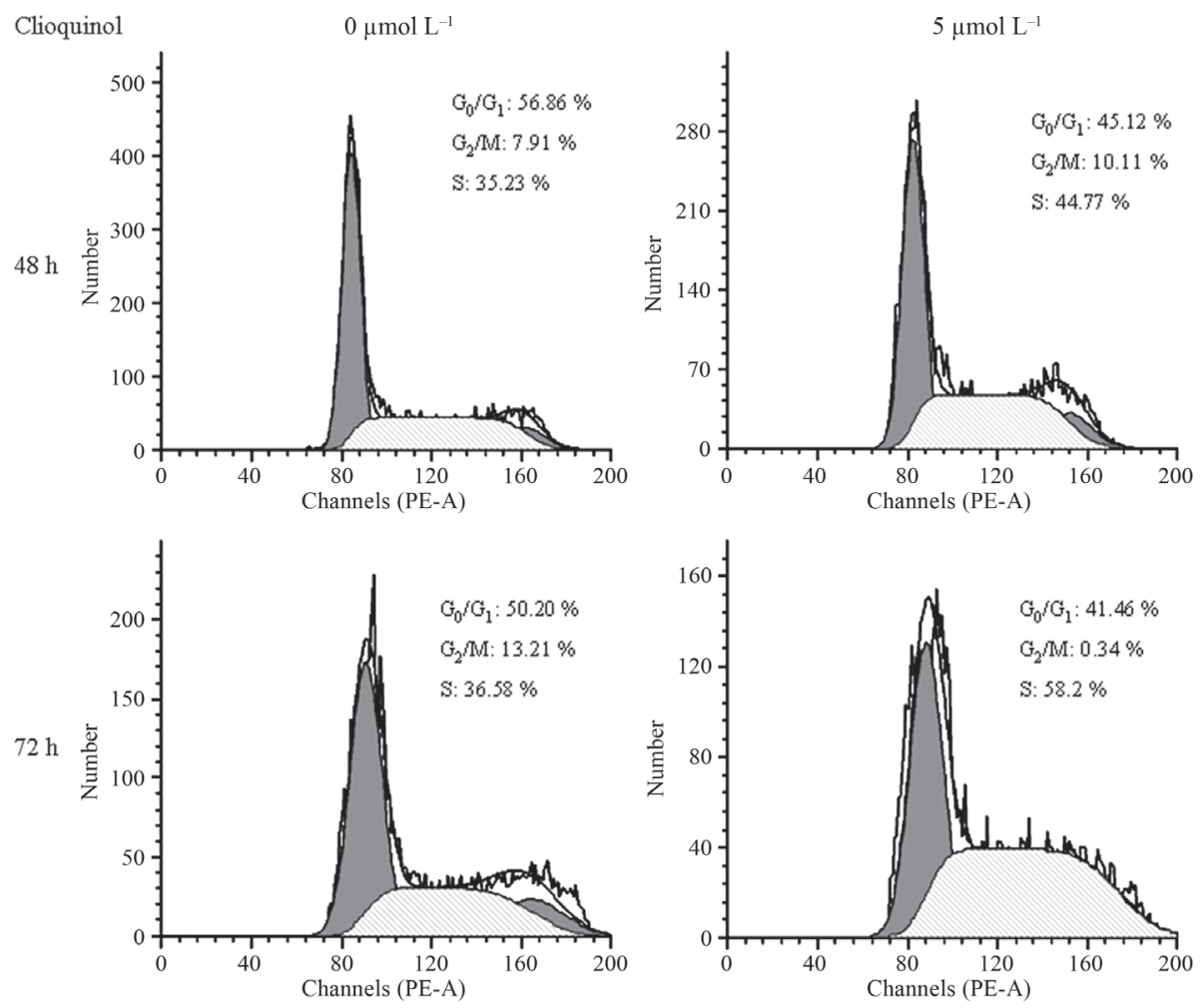

Fig. 3. Clioquinol inhibits cell cycle progression and causes S-phase arrest in SMMC-7721 cells.

\section{Changes of cell cycle related genes $m R N A$ and proteins expression}

In order to determine whether clioquinol was able to regulate cell cycle related gene expression, a real-time PCR experiment was performed in the cell extracts prepared from SMMC-7721 cells. The results showed that clioquinol reduced the levels of cyclin A2, cyclin D1, cyclin E1 and Cdk2 $(p<0.05)$, and increased the levels of p21 and p27 $72 \mathrm{~h}$ after treatment $(p<0.01)$ (Fig. 4). Next, Western blot with specific antibodies was done to detect the changes of the related protein expression levels. We observed a reduction of cyclin A2, cyclin D1, cyclin E1 and Cdk2 and an increase of p21 and p27 after the treatment with clioquinol for $72 \mathrm{~h}$ (Fig. 5). Therefore, clioquinol regulates mRNA and subsequent protein levels of certain cell cycle-related genes to induce the S-phase cell cycle arrest in SMMC7721 cells. 


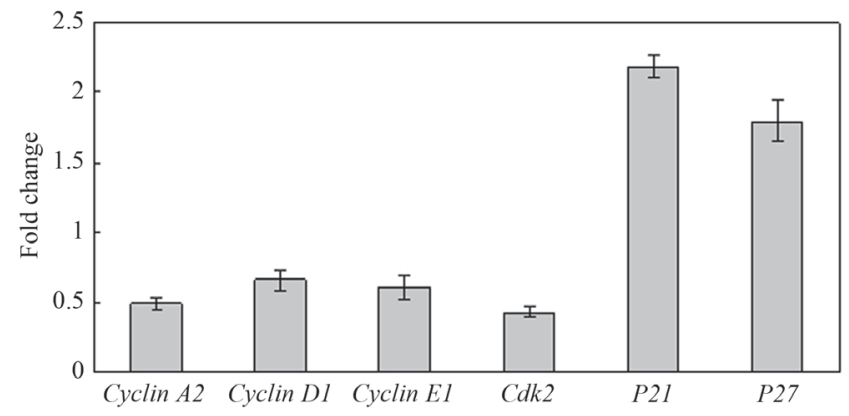

Fig. 4. Assessment of the relative mRNA level of cell cycle related genes after clioquinol treatment in SMMC-7721 cells by real-time PCR.

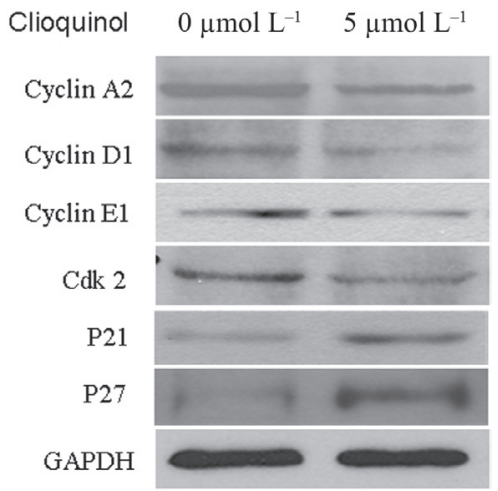

Fig. 5. Western blotting analysis of the expression level of cell cycle related proteins after clioquinol treatment in SMMC-7721 cells.

Clioquinol has been shown to have anticancer activity in several experimental model systems via several different mechanisms of action. For instance, it generates free zinc in lysosomes as a zinc ionophore (17), inhibits NF-kappa B activity $(12,17,18)$, or proteasome activity $(8,9,19)$, induces the cytoplasmic clearance of X-linked inhibitor of apoptosis protein (20), or induces DNA double-strand break and subsequent activation of ATM/p53 signaling (21). The present study firstly demonstrated that clioquinol induced cell-cycle arrest in the S phase in SMMC-7721 hepatoma cells. Furthermore, according to the current data, the $S$ phase arrest in clioquinol treated SMMC-7721 cells was accompanied by the alteration of various cell cycle-related proteins. SMMC-7721 cells treated with clioquinol showed down-regulation of cyclin A2, cyclin D1, cyclin E1 and Cdk2, and up-regulation of p21 and p27. A previous study has reported that the $S$ phase cell-cycle arrest occurs with the loss of Cdk2 activity due to up-regulation of p21 and reduced formation of active complex cyclin E/Cdk2 kinase (22). Yeo et al. (23) suggested that an intra-S-phase checkpoint occurs via phosphorylation of Chk1/2, which leads to degradation of cdc25A and deactivation of cyclin E/A-Cdk2 complex. In this study, up-regulation of p21, p27 and down-regu- 
lation of cyclin E1, cyclin A2 and Cdk2 are considered to be involved in the S-phase arrest induced by clioquinol in SMMC-7721 cells. In addition, clioquinol regulates the mRNA levels of these cell cycle-related genes and results in subsequent protein levels to induce the S-phase cell cycle arrest in SMMC-7721 cells. However, the cell cycle regulation by clioquinol needs to be elucidated further.

\section{CONCLUSIONS}

Our preliminary findings demonstrate for the first time that clioquinol exerts its cell death effects via S-phase cell-cycle arrest in SMMC-7721 hepatoma cells. Additionally, the growth inhibitory effect after exposure to clioquinol is not caused by apoptosis and/or autophagy in SMMC-7721 cells. Although clioquinol exhibits different modes of action in different types of cancer cells, here it suppresses cell cycle progression in the S-phase in SMMC-7721 cells via the p21, p27-cyclin E,A/Cdk2 pathway. Our results strongly suggest that clioquinol should be additionally investigated as a potential novel chemotherapeutic agent for the treatment of some malignant human cancers.

Acknowledgements. - We are grateful to Dr. Deeksha Vishwamitra for her kind assistance in language correction. This work was sponsored by grants from the Natural Science Foundation of Shanghai (15ZR1400200), Shanghai Scientific and Technological Innovation Project (14520720700), State Education Ministry and Fundamental Research Funds for the Central Universities (2232014A3-03, 2232015G1-41 and 222201313010), the Applied Basic Research Project of Promotion Plan for Scientific and Technological Innovation in Qinghai Province (2015-ZJ-703).

\section{REFERENCES}

1. L. Cahoon, The curious case of clioquinol, Nat. Med. 15 (2009) 356-359; DOI: 10.1038/nm0409-356.

2. J. Tateishi, Subacute myelo-optico-neuropathy: clioquinol intoxication in humans and animals, Neuropathology 20 (2000) S20-S24; DOI: 10.1046/j.1440-1789.2000.00296.x.

3. T. Tsubaki, Y. Honma and M. Hoshi, Neurological syndrome associated with clioquinol, Lancet $\mathbf{1}$ (1971) 696-697.

4. L. Helmuth, Neuroscience. An antibiotic to treat Alzheimer's? Science 290 (2000) 1273-1274; DOI: 10.1126/science.290.5495.1273a.

5. X. Mao and A. D. Schimmer, The toxicology of clioquinol, Toxicol. Lett. 182 (2008) 1-6; DOI: 10.1016/j.toxlet.2008.08.015.

6. M. H. Park, S. J. Lee, H. R. Byun, Y. Kim, Y. J. Oh, J. Y. Koh and J. J. Hwang, Clioquinol induces autophagy in cultured astrocytes and neurons by acting as a zinc ionophore, Neurobiol. Dis. 42 (2011) 242-251; DOI: 10.1016/j.nbd.2011.01.009.

7. S. R. Bareggi and U. Cornelli, Clioquinol: review of its mechanisms of action and clinical uses in neurodegenerative disorders, CNS Neurosci. Ther. 18 (2012) 41-46; DOI: 10.1111/j.1755-5949.2010. 00231.x.

8. K. G. Daniel, D. Chen, S. Orlu, Q. C. Cui, F. R. Miller and Q. P. Dou, Clioquinol and pyrrolidine dithiocarbamate complex with copper to form proteosome inhibitors and apoptosis inducers in human breast cancer cells, Breast Cancer Res. 7 (2005) 897-908; DOI: 10.1186/bcr1322.

9. T. Du, G. Filiz, A. Caragounis, P. J. Crouch and A. R. White, Clioquinol promotes cancer cell toxicity through tumor necrosis factor alpha release from macrophages, J. Pharmacol. Exp. Ther. 324 (2008) 360-367; DOI: 10.1124/jpet.107.130377. 
10. M. W. Lee, P. C. Lin and W. C. Tsai, The anti-cancer effects of clioquinol on oral cancer, EACR-23 Poster Sessions/Eur. J. Cancer 50 (2014) S42.

11. O. Afzal, S. Kumar, M. R. Haider, M. R. Ali, R. Kumar, M. Jaggi and S. Baw, A review on anticancer potential of bioactive heterocycle quinolone, Eur. J. Med. Chem. 97 (2015) 871-910; DOI: 10.1016/j. ejmech.2014.07.044.

12. W. Q. Ding, B. Liu, J. L. Vaught, H. Yamauchi and S. E. Lind, Anti-cancer activity of the antibiotic clioquinol, Cancer Res. 65 (2005) 3389-3395; DOI: 10.1158/0008-5472.CAN-04 -3577.

13. X. Mao, X. Li, R. Sprangers, X. Wang, A. Venugopal, T. Wood, Y. Zhang, D. A. Kuntz, E. Coe, S. Trudel, D. Rose, R. A. Batey, L. E. Kay and A. D. Schimmer, Clioquinol inhibits the proteasome and displays preclinical activity in leukemia and myeloma, Leukemia 23 (2009) 585-590; DOI: 10.1038/leu.2008.232.

14. A. D. Schimmer, Y. Jitkova, M. Gronda, Z. Wang, J. Brandwein, C. Chen, V. Gupta, A. Schuh, K. Yee, J. Chen, S. Ackloo, T. Booth, S. Keays and M. D. Minden, A Phase I study of the metal ionophore clioquinol in patients with advanced hematologic malignancies, Clin. Lymphoma Myeloma Leuk. 12 (2012) 330-336; DOI: 10.1016/j.clml.2012.05.005.

15. T. Kanzawa, Y. Kondo, H. Ito, S. Kondo and I. Germano, Induction of autophagic cell death in malignant glioma cells by arsenic trioxide, Cancer Res. 63 (2003) 2103-2108.

16. K. J. Livak and T. D. Schmittgen, Analysis of relative gene expression data using real-time quantitative PCR and the 2-delta delta CT method, Methods 52 (2001) 402-408; DOI: 10.1006/ meth.2001.1262.

17. H. Yu, Y. Zhou, S. E. Lind and W. Q. Ding, Clioquinol targets zinc to lysosomes in human cancer cells, Biochem. J. 417 (2009) 133-139; DOI: 10.1042/BJ20081421.

18. H. Yu, J. R. Lou and W. Q. Ding, Clioquinol independently targets NF kappa B and lysosome pathways in human cancer cells, Anticancer Res. 30 (2010) 2087-2092.

19. D. Chen, Q. C. Cui, H. Yang, R. A. Barrea, F. H. Sarkar, S. Sheng, B. Yan, G. P. Reddy and Q. P. Dou, Clioquinol, a therapeutic agent for Alzheimer's disease, has proteasome-inhibitory, androgen receptor-suppressing, apoptosis-inducing, and antitumor activities in human prostate cancer cells and xenografts, Cancer Res. 67 (2007) 1636-1644; DOI: 10.1158/0008-5472. CAN -06-3546.

20. M. A. Cater and Y. Haupt, Clioquinol induces cytoplasmic clearance of the X-linked inhibitor of apoptosis protein (XIAP): therapeutic indication for prostate cancer, Biochem. J. 436 (2011) 481-491; DOI: 10.1042/BJ20110123.

21. M. Katsuyama, K. Iwata, M. Ibi, K. Matsunob, M. Matsumotob and C. Yabe-Nishimura, Clioquinol induces DNA double-strand breaks, activation of ATM, and subsequent activation of p53 signaling, Toxicology 299 (2012) 55-59; DOI: 10.1016/j.tox.2012.05.013.

22. C. Laezza, S. Pisanti, E. Crescenzi and M. Bifulco, Anandamide inhibits Cdk2 and activates Chk1 leading to cell cycle arrest in human breast cancer cells, FEBS Lett. 580 (2006) 6076-6082; DOI:10.1016/j.febslet.2006.09.074.

23. E. J. Yeo, J. H. Ryu, Y. S. Chun, Y. S. Cho, I. J. Jang, H. Cho, J. Kim, M. S. Kim and J. W. Park, YC-1 induces $\mathrm{S}$ cell cycle arrest and apoptosis by activating checkpoint kinases, Cancer Res. 66 (2006) 6345-6352; DOI: 10.1158/0008-5472.CAN-05-4460. 